\title{
Insights on the origin of the $\mathrm{Tb}_{5} \mathrm{Ge}_{4}$ Magnetocaloric effect
}

\author{
J.H. Belo ${ }^{1)}$, M. B. Barbosa ${ }^{1)}$, A. L. Pires ${ }^{2)}$, R. M. Costa ${ }^{1)}$, J.G.V. Teixeira ${ }^{1)}$, J. Silva ${ }^{1)}$, P.A Algarabel ${ }^{3,5)}$, \\ C.Magen $^{4,5,6)}$, L. Morellon ${ }^{5,6)}$, J.S. Amaral ${ }^{1,7)}$, U. Zeitler ${ }^{8)}$, G. Veerendra ${ }^{8)}$, A. M. dos Santos ${ }^{9)}$, Y. Ren ${ }^{10)}$, \\ M.R. Ibarra ${ }^{5,6)}$ J.P. Araújo ${ }^{1, \text { a) }}$ A. M. Pereira ${ }^{1, b)}$
}

In this report the magnetic, atomic structures and spin-lattice coupling have been thoroughly studied through high magnetic field magnetometry, Synchrotron X-ray diffraction under applied magnetic field and magnetostriction measurements in the $\mathrm{Tb}_{5} G e_{4}$ compounds. A field induced phase transition from an antiferromagnetic towards a ferromagnetic ordering was confirmed but with absence of structural transformation. This absence has been confirmed experimentally through synchrotron x-ray diffraction under applied field (up to 30T). Moreover, this absence was explained via a thermodynamic free energy model: first principles calculations determined a large energy gap $(\Delta \mathrm{E}=0.65 \mathrm{eV})$ between the two possible structures, $\mathrm{O}(\mathrm{I})$ and $\mathrm{O}(\mathrm{II})$. From magnetic and structural properties, a H-T phase diagram has been proposed for $\mathrm{Tb}_{5} \mathrm{Ge}_{4}$. Finally it was observed a large magnetostriction (up to $600 \mathrm{ppm}$ ) induced by $\Delta \mathrm{H}=7 \mathrm{~T}$.

Keywords: Magnetocaloric effect, Phase diagram, Magnetic phase transitions, Structure of crystalline solids.

PACS: $75.30 . \mathrm{Sg}$, 81.30.Bx, 75.30.Kz, 61.66.-f 


\section{INTRODUCTION}

The magnetocaloric effect (MCE) is known for over 100 years and since then it has been used to achieve ultralow temperatures [1,2]. However, the interest in MCE has been recently boosted due to the discovery of new materials displaying giant MCE (GMCE) near room temperature, which allow its use on home fridges or air conditioning to become closer to reality [3-6]. In the last seventeen years more than 42 MCE-based prototypes were developed [7]. The magnetic refrigeration technology is environmentally friendly (prevents the use of CFCs) and shows larger efficiency, reliability and lower noise [8-10], turning it the most promising substitute to gascompression/expansion technology [11]. Among the set of compounds with GMCE, we highlight the $\mathrm{R}_{5}(\mathrm{Si}, \mathrm{Ge})_{4}$ family, which have compounds presenting the highest MCEs and the widest temperature range where the GMCE is exhibited (20-350 K), when the $\mathrm{R}$ element or the $\mathrm{Si} / \mathrm{Ge} / \mathrm{T}$ ( $\mathrm{T}$ - transition metal) concentration is varied [12-14]. The most prominent feature of these compounds, showing GMCE, is the fact that most of them undergo a firstorder structural and magnetic phase transition, leading to a giant magnetic field-induced entropy change across their ordering temperature [15]. There are three main atomic structures adopted by these materials: Orthorhombic O(II) (Pnma space group), Monoclinic M (P112/a space group) and Orthorhombic O(I) (Pnma space group). Their detailed characterization is thoroughly described in several reports such as[16-19], here only their main features will be mentioned. They can be characterized as stacks of rigid slabs composed by 5 atomic layers each $[\mathrm{Si}(\mathrm{Ge}) / \mathrm{R} / \mathrm{R}-\mathrm{Si}(\mathrm{Ge}) / \mathrm{R} / \mathrm{Si}(\mathrm{Ge})]$. Between slabs, in the interslab region, there are $\mathrm{Si}$ and $\mathrm{Ge}$ atoms which may form covalent bonds, hence connecting two adjacent rigid slabs. The presence of these bonds is one of the features which characterizes each structure: $\mathrm{O}(\mathrm{II})$ does not have any bond formed whereas $\mathrm{M}$ has half of them and $\mathrm{O}(\mathrm{I})$ has all bonds formed. This justifies the structures volume: $\mathrm{O}$ (II) has the highest volume followed by $\mathrm{M}$ and $\mathrm{O}$ (I), $\mathrm{V}_{\mathrm{O} \text { (II) }}$ $>\mathrm{V}_{\mathrm{M}}>\mathrm{V}_{\mathrm{O}(\mathrm{I})}$. Microscopically, the structural transition is described as an opposite displacement of two adjacent rigid slabs. This movement leads to a break/formation of the covalent bonds between the $\mathrm{Si}-\mathrm{Ge}$ atoms at the interslab region and the consequent transformation into a different structure. Although this transition occurs both with temperature and magnetic field variation in several $R_{5}(\mathrm{Si}, \mathrm{Ge})_{4}$ specimens, in $\mathrm{Gd}_{5} G e_{4}$ the magneto-structural transition is never induced by temperature variation, but only occurs under an external applied magnetic field 
and/or external pressure [20-26], for $\mathrm{T}<60 \mathrm{~K}$. This induced magneto-structural transition consists in a structural change from $\mathrm{Sm}_{5} \mathrm{Ge}_{4}$-type [O(II)] to a $G d_{5} S i_{4}$-type structure [O(I)], i.e. formation of covalent bonds between GeGe atoms at the interslab $[\mathrm{O}(\mathrm{I})]$. In absence of magnetic field, $G d_{5} G e_{4}$ presents a purely second order phase transition at $\mathrm{T}_{\mathrm{N}} \sim 125 \mathrm{~K}$, changing from paramagnetic $(\mathrm{PM})$ to antiferromagnetic (AFM) on cooling [27]. Furthermore, this specific compound presents other important magnetic properties such as strong magnetic anisotropy [28], short-range magnetic correlations in the PM matrix [29,30], magnetic glass state [31,32], spin flip transition [23,27,33]. Paudyal et al., used first principles calculations to understand the magnetic and crystallographic structures of $G d_{5} G e_{4}[22,34]$ and reported that the anti-symmetric interslab Ge-Ge covalent bonds lead to long-range FM-order in the $\mathrm{O}(\mathrm{I})$ phase, while only short-range FM-order is supported in the O(II) phase. The free energy gap (at $0 \mathrm{~K}$ ) between the two Orthorhombic structures (assuming FM interactions) involved in the transition was estimated to be $\Delta \mathrm{E} \sim 0.362 \mathrm{eV} /$ cell[22], pointing the energy barrier height that must be overcome in order to promote the transition, that in this case is only achieved by applying a strong enough magnetic field or hydrostatic pressure. Due to such exotic behavior, in particular its exclusive magnetic field structural transition, the end member $G d_{5} G e_{4}$ has attracted major interest in the last few years.

Since the discovery of the $R_{5}\left(S i_{x} G e_{1-x}\right)_{4}$, the $\mathrm{R}=G d$ and $T b$ families have been studied in parallel due to their similar properties, a consequence of their similar magnetic and structural phase diagram. This comparison method has been fruitful for deepen our knowledge on both compound families. The $G d_{5} G e_{4}$ neighbour, $T b_{5} G e_{4}$, was firstly studied by P. Schobinger Papamantellos [35] in 1976, and now recently detailed magnetic and structural studies were performed by C. Ritter et al.[36] revealing that $T b_{5} G e_{4}$ compound stabilizes in the [PM, $\mathrm{O}(\mathrm{II})]$ state at room temperature and undergoes a second-order magnetic transition at $\mathrm{T}_{\mathrm{N}} \sim 95 \mathrm{~K}$ changing from a $\mathrm{PM}$ to a canted AFM state, a similar transition as observed in $G d_{5} G e_{4}$. Another magnetic transition, also of second order, is observed at $\mathrm{T}_{\mathrm{SR}} \sim 55 \mathrm{~K}$ related to a small rotation on the magnetic moments direction (spin reorientation transition), as also occurs for the GMCE compound $\mathrm{Tb}_{5} \mathrm{Si}_{2} \mathrm{Ge}_{2}$ [37]. Despite these important contributions, $T b_{5} G e_{4}$ has been the focus of few studies which have left unclear, for instance, if a similar exclusive magnetic field 
structural transition also occurs in this system or not. Hence, the main purpose of this work is to understand more deeply the coupling between the atomic and magnetic structures in $T b_{5} G e_{4}$ compound.

\section{EXPERIMENTAL DETAILS}

Polycrystalline specimen of $T b_{5} G e_{4}$ was synthesized by arc melting of stoichiometric mixtures of high-purity $T b$ (99.9 wt.\%) and Ge (99.9999 wt.\%). Weight losses during melting were negligible and therefore the initial compositions were assumed unchanged. The crystallographic structure was determined by Rietveld refinement of the X-ray diffraction patterns using the software package FULLPROF [38]. Magnetization experiments were performed in a commercial (Quantum Design) Superconducting Quantum Interference Device (SQUID) magnetometer in the temperature range 5-300 K and magnetic fields up to $50 \mathrm{kOe}$ and Vibrating Sample Magnetometer (VSM) in the temperature range 5-300 K and magnetic fields up to $100 \mathrm{kOe}$ or $300 \mathrm{kOe}$ that were achieved using an extraction magnetometer in a Bitter magnet, HFML (Nijmegen, NL). Magnetostriction measurements were performed with the strain gauge technique from 4 to $300 \mathrm{~K}$ and using PPMS in Zaragoza University. The X-ray diffraction synchrotron (XRS) experiments were performed on the 11-D-C instrument $(\lambda=$ $0.1066 \AA$ ) at the Advanced Photon Source at Argonne National Laboratory (Argonne, USA) under applied magnetic fields up to $60 \mathrm{kOe}$ at $\mathrm{T}=5 \mathrm{~K}$. ab-initio calculations were performed using the Wien2K code13 via the augmented plane wave method with local orbitals $(\mathrm{APW}+\mathrm{lo})$ approach in order to estimate the free energies of both Orthorhombic $\mathrm{O}(\mathrm{I})$ and $\mathrm{O}(\mathrm{II})$ structures.

\section{EXPERIMENTAL RESULTS AND DISCUSSION}

\section{A. Magnetic characterization}

In Fig. 1 the temperature dependence of the magnetization in the 5-300 $\mathrm{K}$ range is presented, confirming that at room temperature the sample is in the PM state. On cooling, a magnetic second order phase transition (SOPT) occurs at $\mathrm{T}_{\mathrm{N}} \sim 92 \mathrm{~K}$ changing to a canted AFM state. At lower temperatures, a second magnetic order-order transition occurs at $\mathrm{T}_{\mathrm{SR}} \sim 55 \mathrm{~K}$, due to the rearrangement of the magnetic moments [40]. The temperature dependence of the inverse magnetic susceptibility, $\chi^{-1}(\mathrm{~T})$, displays an anomaly on cooling at a critical temperature 
$\mathrm{T}_{\mathrm{G}}$, where a deviation from the Curie-Weiss law is observed (inset Fig. 1 a)), due to the emergence of short range magnetic clusters, the so called Griffiths-like phase [30]. Using the Curie-Weiss law above $\mathrm{T}_{\mathrm{G}}, \Theta_{\mathrm{P}} \sim 61 \mathrm{~K}$ and $\mu_{\text {eff }}$ $\sim 9.6 \mu_{\mathrm{B}} / \mathrm{T}_{\mathrm{b}}$ were estimated, which is in fair agreement with the theoretical prediction for a $T b^{3+}$ free ion $\left(\mu_{\mathrm{eff}}=\right.$ $9.72 \mu_{\mathrm{B}} / \mathrm{T}_{\mathrm{b}}$ ). Furthermore, the magnetization response to high magnetic fields application is represented in Fig. 1 b). For all curves, a drastic magnetization increase is observed at a critical field value, $\mathrm{H}_{\mathrm{C}}$, which lies in the [4.5, 6.5] $\mathrm{T}$ field range. For $\mathrm{H}>\mathrm{H}_{\mathrm{C}}$, the magnetization continues to increase, but more steadily, whereas for even higher fields $(\mathrm{H}>12 \mathrm{~T})$ magnetization approaches saturation. When decreasing the magnetic field towards the zero value, the magnetization behavior follows a different path (with higher magnetization values) which leads to the appearance of magnetic hysteresis, particularly noticeable in the $\mathrm{T}=68 \mathrm{~K}$ isotherm. The drastic magnetization increase clearly corresponds to a magnetic phase transition from a lower magnetization state towards a high magnetization one. Considering the information of the different magnetic states extracted from the $\mathrm{M}(\mathrm{T})$ curve and from previous works $[35,40]$, this field-induced magnetic transition occurs from a low field canted AFM towards a high-field ferromagnetic ordering (FM) - hence being an order-order phase transition occurring at a certain field $\mathrm{H}_{\mathrm{C}}$. Such behavior has also been observed in the $G d$ counterpart [41]. Low temperature isotherms, $\mathrm{T}$ $<50 \mathrm{~K}$, exhibit an extra bump when the magnetic field approaches $\mathrm{H}_{\mathrm{C}}$. Such feature is associated with a spin reorientation mechanism, responsible for the transition from an AFM1 to an AFM2 state, similarly as the spinflop behavior demonstrated by $G d_{5} G e_{4}[23,27,33,42]$. At $\mathrm{H}=7 \mathrm{~T}$, the $\mathrm{T}=4.4 \mathrm{~K}$ isotherm curve presents a magnetization value $\left(\mu_{\mathrm{S}}=8.3 \mu_{\mathrm{B}} / \mathrm{Tb}\right)$ that is still lower than the expected theoretical one $\left(\mathrm{gJ}=9 \mu_{\mathrm{B}} / \mathrm{Tb}^{3+}\right)$, revealing the presence of small canting effects in the $T b^{3+}$ ions even after the drastic magnetization increase transition. Above $12 \mathrm{~T}$, the saturated values $\left(\mu_{\mathrm{S}}=8.9 \mu_{\mathrm{B}} / \mathrm{T}_{\mathrm{b}}\right)$ are close to the expected theoretical one, revealing the presence of an almost perfect collinear alignment of the $\mathrm{Tb}^{3+}$ ions. These findings are in accordance with neutron diffraction results $[35,40][26,32]$ if it is only considered a rotation of the macro magnetic moment of the rigid slab to align with the applied magnetic field. Hence, sustaining that this metamagnetic-like transition is described by a cooperative rotation of the macro magnetic moment of the rigid slab, i.e. spin flip of the total magnetic moment of each rigid slab initially in the antiparallel configuration (AFM state) towards a parallel configuration (FM state). However the same spin canted moment's configuration within the rigid slab is preserved even at the 
end of this transition. Afterwards, the effect of increasing further the magnetic field is to rotate each $T b$ atom magnetic moment inside the rigid slab towards the magnetic field direction, reaching the theoretical saturation value.

\section{B. Structural characterization}

In order to better understand the magnetic and structural coupling occurring in this compound, magnetostriction (MS) experiments have been performed in the $[0,9] \mathrm{T}$ magnetic field range and $[4,100] \mathrm{K}$ temperature interval, as can be observed in Fig. 2 a). A relatively large variation of the MS (up to $600 \mathrm{ppm}$ ) is observed, associated with the magnetic transition since it occurs at the same critical fields as the ones observed in the $\mathrm{M}(\mathrm{H})$ curves (see Fig. 1 b)). Thus, evidencing the strong coupling between the magnetic and elastic properties, typical of the $R_{5}(S i$, $\mathrm{Ge})_{4}$ compounds [15,43-45]. Indeed, the MS data behavior portrayed in Fig. 2 a) shows an initial decrease before $\mathrm{H}_{\mathrm{C}}$ followed by a linear increase towards a classical positive MS [46]. The highest magnetostriction value measured in this sample $(600 \mathrm{ppm})$ is approximately 4 to 10 times lower than the maximum magnetostriction values observed in first-order transition systems, such as $G d_{5} S i_{1.8} G e_{2.2}$ (4500 ppm) [15], $G d_{5} S i_{2} G e_{2}(6000 \mathrm{ppm})$ [46] and $\mathrm{Tb}_{5} S i_{2.2} G e_{1.8}(2000 \mathrm{ppm})$ [47] and even lower than the expected values (10000 ppm) lattice parameter change from x-ray diffraction under magnetic applied field) for $G d_{5} G e_{4}$ [48]. Simultaneously, the $T b_{5} G e_{4}$ observed saturation magnetostriction value is close to the one obtained for pure $\mathrm{Tb}[49]$ and for $T b_{5} S i_{2.2} G e_{1.8}$ [47] and $\mathrm{Tb}_{5} \mathrm{Si}_{2} \mathrm{Ge}_{2}$ [50] compounds at temperatures below their critical first-order transition temperature. Hence, in order to clarify the nature of the MS response, XRS experiments under two applied magnetic fields $(\mathrm{H}=0$ and 69 kOe) were performed at $\mathrm{T}=5 \mathrm{~K}$. The two spectra are shown in Fig. 2 b). No relevant changes occurred when a 69 kOe magnetic field was applied in comparison with the zero field spectra: the main reflections occur at the same positions and the relative intensities remain approximately constant, only smooth changes occur in the intensity at $\theta \sim 3.5^{\circ}$ and $3.7^{\circ}$ reflections as is highlighted in the Fig. 2 b) inset. Such results demonstrate that a 69 kOe magnetic field is not able to induce a structural transition at $\mathrm{T}=5 \mathrm{~K}$, in contrast to the $G d_{5} G e_{4}$ case [48]. 


\section{Nature of the magnetic transition}

To better understand the obtained results, an estimation of the temperature dependence of the free energies was performed for each $\mathrm{Tb}_{5} G e_{4}$ structure: $\mathrm{O}(\mathrm{II})$ and $\mathrm{O}(\mathrm{I})$ - where the latter was considered the most probable high field structure, in accordance with the $G d_{5} G e_{4}$ compound. The approach followed was the one described in previous works [43,51]: ab-initio calculations were performed in order to estimate the free energies of the two structures at $\mathrm{T}=0 \mathrm{~K}$ and the equation:

$$
F(T, H)=U-T S=-T S^{\text {lattice }}-H g J \sigma(T, H)-\frac{3}{2} \frac{J}{J+1} k_{B} T_{C} \sigma(T, H)^{2}-T S^{m}+U_{0}
$$

where $S^{\text {lattice }}$ is obtained from the Debye model, $\sigma(T, H)$ is the reduced magnetization that depends on temperature and applied magnetic field, the second term is the interaction energy between a localized magnetic moment and $\mathrm{H}$ (Zeeman term), the third one is the magnetic ion-ion exchange interaction energy, the fourth term is derived from the Mean Field Approximation (MFA) and the last term $\left(\mathrm{U}_{0}\right)$ is composed by two parts: one is obtained by firstprinciples calculations (lattice, electronic and magnetic contributions at $0 \mathrm{~K}$ ) summed with $+3 / 2 \mathrm{~J} /(\mathrm{J}+1) \mathrm{k}_{\mathrm{B}} \mathrm{T}_{\mathrm{C}}$ (to remove the third term value at $0 \mathrm{~K}$ ). The ordering temperatures, $\mathrm{T}_{\mathrm{C}}$, considered were $189 \mathrm{~K}$ and $92 \mathrm{~K}$ for structure $\mathrm{O}(\mathrm{I})$ and $\mathrm{O}(\mathrm{II})$, based on the linear extrapolation of the Curie temperature from Si-rich region of the magnetic and structural phase diagram and from the $\mathrm{M}(\mathrm{T})$ curve, respectively [43,51].

In order to determine the $\mathrm{T}=0 \mathrm{~K}$ free energy difference between the $\mathrm{O}(\mathrm{I})$ and $\mathrm{O}(\mathrm{II})$ phases, ab-initio calculations were performed using Wien2k code, via the augmented plane wave method with local orbitals $(\mathrm{APW}+\mathrm{lo})$ approach and considering the intra-atomic Coulomb correlation term $\mathrm{U}(\mathrm{LDA}+\mathrm{U})$, with $\mathrm{U}$ and $\mathrm{J}$ assuming the values 6.7 and $0.7 \mathrm{eV}$, respectively, as previously used in $\mathrm{Tb}_{5} \mathrm{Si}_{2} \mathrm{Ge}_{2}$ similar calculations [44,52]. These calculations assumed a ferromagnetic spin polarization of $T b$ atoms and kept the structure fixed. The estimated energy difference between the two structures was was $\Delta \mathrm{F} \equiv \mathrm{F}_{\mathrm{O}(\mathrm{I})}-\mathrm{F}_{\mathrm{O}(\mathrm{II})}=0.65 \mathrm{eV} / \mathrm{cell}$. Although the $\mathrm{O}$ (II) magnetic order is AFM and not FM as considered, the total free energy is expected to be very similar for the AFM ordering, with a variation lower than $10 \%$, as was estimated in similar calculation in the $G d_{5} G e_{4}$ 
compound [22]. Regarding Eq. 1, the $\mathrm{S}^{\text {lattice }}$ term also has a contribution smaller than $10 \%$ of the total free energy estimation, and hence one can neglect it when the free energies temperature dependence is estimated [43]. The temperature dependence of both free energies is estimated and plotted in Fig. 3. As can be seen the O(II) free energy is the lowest one up to $200 \mathrm{~K}$ and no crossing with the $\mathrm{O}(\mathrm{I})$ free energy curve is observed. As explained in previous works $[43,53]$, the magnetic field effect on these curves is to smear them along the temperature interval, where the smearing is larger for the free energy of the structure with lower ordering temperature (O(II)). Nevertheless, according to this thermodynamics model, the free energies difference is too large ( $\sim 40 \%$ larger than in the $G d_{5} G_{4}[22]$ and 4 times higher than in $\mathrm{Tb}_{5} \mathrm{Si}_{2} \mathrm{Ge}_{2}$ [43] compounds) to be overcome by an experimental available magnetic field. Hence, the ab-initio calculations and the free energies estimation analysis corroborate with the XRS measurements and analysis, suggesting the conclusion that no available magnetic field is able to induce the $\mathrm{O}(\mathrm{II})-\mathrm{O}(\mathrm{I})$ structural transition, in the whole temperature interval considered, in contrast to what occurs in $G d_{5} G e_{4}$. In the light of these results both the magnetic hysteresis observed in the $\mathrm{M}(\mathrm{H})$ curves observed in Fig. $1 \mathrm{~b})$ and the large MS values represented in Fig. 2 a) have a pure magnetic nature. The MS data behavior portrayed in Fig. 2 a) can be explained considering two different contributions. Firstly, the initial decrease near $\mathrm{H}_{\mathrm{C}}$ is normally attributed to grain boundary effects $[49,54]$. Secondly, the linear increase is caused by the rotation of the magnetic moments that results in the classical positive MS [46,54], reaching saturation, when all domains have their magnetic moments aligned with the field direction. Above $\mathrm{T}_{\mathrm{N}},(\mathrm{T}=100 \mathrm{~K}$ curve) the MS presents a quadratic contribution, typical of the PM state caused by the rotation of the magnetic moments that results in the classical positive MS [49]. In fact, the measured MS curves show very similar behavior with the ones exhibited by pure $\mathrm{Tb}[49]$. 


\section{H-T phase diagram and the magnetocaloric effect}

The $\mathrm{Tb}_{5} \mathrm{Ge}_{4}$ proposed (H-T) phase diagram is presented in Fig. 4 a). Two AFM phases are expected at low temperatures, the AFM1 $(120<\mathrm{T}<55 \mathrm{~K})$ and AFM2 $(\mathrm{T}<55 \mathrm{~K})$ states. At higher fields, $\mathrm{H}_{=} \mathrm{H}_{\mathrm{C}}\left(\mathrm{H}_{\mathrm{C}} \sim 4 \mathrm{~T}\right.$ at 4 $\mathrm{K})$, a magnetic order-order transitions displayed at low temperatures $(\mathrm{T}<100 \mathrm{~K})$ with the system changing from the AFM2 or AFM1 states to the FM, but retaining the same $\mathrm{O}(\mathrm{II})$ structural matrix. As field continues to increase a complete collinear alignment between all $\mathrm{Tb}$ atoms is expected at the saturation regime. Furthermore, the field invariance of the lower temperature peak of $\triangle \mathrm{SM}(\mathrm{T})$ curve [40] suggests the vertical behavior of the AFM1AFM2 boundary. In comparison with the $\mathrm{H}-\mathrm{T} \mathrm{Gd}_{5} \mathrm{Ge}_{4}$ phase diagram the major differences observed here are the presence of a second Antiferromagnetic state (AFM2) and an unique temperature and field independent $\mathrm{O}$ (II) atomic structure.

\section{CONCLUSIONS}

In this work $\mathrm{Tb}_{5} \mathrm{Ge}_{4}$ was thoroughly studied through high magnetic field magnetometer, magnetostriction measurements, synchrotron x-ray diffraction under applied magnetic field and first principles calculations. It was observed that, similarly to $\mathrm{Gd}_{5} \mathrm{Ge}_{4}$ compound, an almost completely collinear ferromagnetic state is induced with strong enough magnetic fields $(\mathrm{H}>9 \mathrm{~T})$, where the magnetic ordering changes progressively from the AFM towards the FM configuration with increasing field. Furthermore, magnetostriction measurements revealed that the AFM-FM transition is accompanied by a large magnetostriction $\sim 600 \mathrm{ppm}$. X-ray synchrotron experiments have shown that $T b_{5} G e_{4}$ does not undergo a structural transition at $\mathrm{T}=5 \mathrm{~K}$, and thus proving the absence of an induced magnetostructural transition, in contrast to the observed in $G d_{5} G e_{4}$. The absence of such a structural transition was explained in terms of ab-initio calculations, which showed that there is a large and insurmountable 
energy gap between the $\mathrm{O}(\mathrm{II})$ and $\mathrm{O}(\mathrm{I})$ structures. Despite causing the reduction of the magnetic responses, such as the magnetocaloric and the magnetostrictive effects, the absence of structural transition represents a favorable feature in future applications since it is a sign of better mechanical stability and consequently longer lifetime.

\section{ACKNOWLEDGEMENTS}

Work partially supported by the projects FEDER/POCTI n0155/94, PTDC/CTM/NAN/5414/2014 from Fundação para a Ciência e Tecnologia (FCT), Portugal. J.H.B. Silva and M. B. Barbosa thanks FCT for the Grant SFRH/BD/88440/2012 and SFRH/BD/97591/2013, respectively. C.M. acknowledges the support of the Fundación ARAID. This work was supported by the Spanish Ministry of Science (through Project Nos. MAT2014- 51982-C2-R and C1-R, including FEDER funding) and the Aragon Regional government (Project No. E26). Use of the Advanced Photon Source at Argonne National Laboratory was supported by the U.S. Department of Energy, Office of Science, Offce of Basic Energy Sciences, under Contract No. DE-AC02-06CH11357. Use of the High Magnetic Field Laboratory at Nijmegen was supported by contract no: NMA01-111.

\section{REFERENCES}

[1] A. Smith, Eur. Phys. J. H. 38 (2013) 507.

[2] R.D. Shull, R.D. McMichael, J.J. Ritter, Nanostructured Mater. 2 (1993) 205.

[3] K.H.J. Buschow, F.R. De Boer, O. Tegus, E. Bru, Nature. 415 (2002) 150.

[4] V.K. Pecharsky, K.A. Gschneidner, Phys. Rev. Lett. 78 (1997) 3.

[5] B.G. Shen, J.R. Sun, F.X. Hu, H.W. Zhang, Z.H. Cheng, Adv. Mater. 21 (2009) 4545.

[6] X. Xu, T. Kanomata, R. Kainuma, Acta Mater. 79 (2014) 159.

[7] L.T. Kuhn, N. Pryds, C.R.H. Bahl, A. Smith, J. Phys. Conf. Ser. 303 (2011) 12082.

[8] K.A. GschneidnerJr, V.K. Pecharsky, A.O. Tsokol, Reports Prog. Phys. 68 (2005) 1479.

[9] W. Choe, G.J. Miller, J. Meyers, S. Chumbley, A.O. Pecharsky, Chem. Mater. 15 (2003) 1413.

[10] K.A. Gschneidner, V.K. Pecharsky, Int. J. Refrig. 31 (2008) 945.

[11] D.R. Brown, J.A. Dirks, N. Fernandez, T.B. Stout, Richland, Washington, n.d. 
[12] V. Franco, J.S. Blázquez, B. Ingale, a Conde, Annu. Rev. Mater. Res. 42 (2012) 305.

[13] J. Yao, P. Lyutyy, Y. Mozharivskyj, Dalton Trans. 40 (2011) 4275.

[14] G.J.K.A. Pecharsky V.K., Appl. Phys. Lett. 70 (1997) 3299.

[15] F.A., L. Morellon, P. A. Algarabel, M. R. Ibarra, J. Blasco, B. Garcia-Landa, Z. Arnold, Phys. Rev. B. 58 (1998) 721.

[16] A. Gschneidner Jr., V.K. Pecharsky, A.O. Tsokol, Reports Prog. Phys. 68 (2005) 1479.

[17] W. Choe, A.O. Pecharsky, M. Warle, G.J. Miller, Inorg. Chem. 42 (2003) 8223.

[18] Y.C. Tseng, D. Haskel, J.C. Lang, Y. Mudryk, V.K. Pecharsky, K.A. Gschneidner, J. Appl. Phys. 103 (2008) 07B301.

[19] Y. Mudryk, V.K. Pecharsky, K.A.J. Gschneidner, Handbook on the Physics and Chemistry of Rare Earths Includind Actinides (Vol. 44), North Holland, 2014.

[20] M.K. Chattopadhyay, M.A. Manekar, A.O. Pecharsky, V.K. Pecharsky, K.A. Gschneidner, J. Moore, G.K. Perkins, Y. V. Bugoslavsky, S.B. Roy, P. Chaddah, L.F. Cohen, Phys. Rev. B - Condens. Matter Mater. Phys. 70 (2004) 1.

[21] V.K. Pecharsky, A.P. Holm, K.A. Gschneidner, R. Rink, Phys. Rev. Lett. 91 (2003) 197204.

[22] D. Paudyal, V.K. Pecharsky, K.A. Gschneidner Jr, B.N. Harmon, Phys. Rev. B. 75 (2007) 94427.

[23] Z.W. Ouyang, V.K. Pecharsky, K.A. Gschneidner, D.L. Schlagel, T.A. Lograsso, Phys. Rev. B - Condens. Matter Mater. Phys. 76 (2007) 1.

[24] Z. Ouyang, H. Nojiri, S. Yoshii, G. Rao, Y. Wang, V. Pecharsky, K. Gschneidner, Phys. Rev. B. 77 (2008) 1 .

[25] Y. Mudryk, D. Paudyal, V.K. Pecharsky, K.A. Gschneidner, S. Misra, G.J. Miller, Phys. Rev. Lett. 105 (2010) 66401.

[26] S.B. Roy, P. Chaddah, V.K. Pecharsky, K.A. Gschneidner Jr., Acta Mater. 56 (2008) 5895.

[27] E.M. Levin, K.A. Gschneidner, T.A. Lograsso, D.L. Schlagel, V.K. Pecharsky, Phys. Rev. B - Condens. Matter Mater. Phys. 69 (2004) 2.

[28] Z. Ouyang, V. Pecharsky, K. Gschneidner, D. Schlagel, T. Lograsso, Phys. Rev. B. 74 (2006) 24401.

[29] Z.W. Ouyang, V.K. Pecharsky, K.A. Gschneidner, D.L. Schlagel, T.A. Lograsso, Phys. Rev. B - Condens. Matter Mater. Phys. 74 (2006) 1.

[30] A.M. Pereira, L. Morellon, C. Magen, J. Ventura, P.A. Algarabel, M.R. Ibarra, J.B. Sousa, J.P. Araújo, Phys. Rev. B. 82 (2010) 172406.

[31] M.K. Chattopadhyay, S.B. Roy, K. Morrison, J.D. Moore, G.K. Perkins, L.F. Cohen, K. a. Gschneidner jr., V.K. Pecharsky, EPL (Europhysics Lett. 83 (2008) 57006.

[32] S.B. Roy, M.K. Chattopadhyay, P. Chaddah, J.D. Moore, G.K. Perkins, L.F. Cohen, K.A. Gschneidner, V.K. Pecharsky, Phys. Rev. B. 74 (2006) 12403. 
[33] L. Tan, A. Kreyssig, S. Nandi, S. Jia, Y.B. Lee, J.C. Lang, Z. Islam, T.A. Lograsso, D.L. Schlagel, V.K. Pecharsky, K.A. Gschneidner, P.C. Canfield, B.N. Harmon, R.J. McQueeney, A. I. Goldman, Phys. Rev. B. 77 (2008) 64425.

[34] D. Paudyal, V.K. Pecharsky, K.A. Gschneidner Jr, J. Phys. Condens. Matter. 20 (2008) 235235.

[35] Penelope Schobinger-Papamantellos, J. Phys. Chem. Solids. 39 (1978) 197.

[36] L. Morellon, C. Magen, P.A. Algarabel, M.R. Ibarra, C. Ritter, Appl. Phys. Lett. 79 (2001) 1318.

[37] J.P. Araújo, A.M. Pereira, M.E. Braga, R.P. Pinto, J.M. Teixeira, F.C. Correia, J.B. Sousa, L. Morellon, P.A. Algarabel, C. Magen, M.R. Ibarra, J. Phys. Condens. Matter. 17 (2005) 4941.

[38] J. Rodriguez-Carvajal, Phys. B. 192 (1993) 55.

[39] L. Caron, Z.Q. Ou, T.T. Nguyen, D.T. Cam Thanh, O. Tegus, E. Bruck, J. Magn. Magn. Mater. 321 (2009) 3559 .

[40] C. Ritter, L. Morellón, P.A. Algarabel, C. Magen, M.R. Ibarra, Phys. Rev. B. 65 (2002) 94405.

[41] H. Tang, V.K. Pecharsky, K.A. Gschneidner, A.O. Pecharsky, Phys. Rev. B. 69 (2004) 64410.

[42] L. Tan, a. Kreyssig, S. Nandi, S. Jia, Y. Lee, J. Lang, Z. Islam, T. Lograsso, D. Schlagel, V. Pecharsky, K. Gschneidner, P. Canfield, B. Harmon, R. McQueeney, a. Goldman, Spin-flop transition in Gd5Ge4 observed by x-ray resonant magnetic scattering and first-principles calculations of magnetic anisotropy, Phys. Rev. B. 77 (2008) 64425. doi:10.1103/PhysRevB.77.064425.

[43] A.M. Pereira, E. Kampert, J.M. Moreira, U. Zeitler, J.H. Belo, C. Magen, P.A. Algarabel, L. Morellon, M.R. Ibarra, J.N. Gonçalves, J.S. Amaral, V.S. Amaral, J.B. Sousa, J.P. Araújo, Appl. Phys. Lett. 99 (2011) 132510.

[44] J.H. Belo, A.M. Pereira, J.P. Araújo, C. de la Cruz, A.M. dos Santos, J.N. Gonçalves, V.S. Amaral, L. Morellon, M.R. Ibarra, P.A. Algarabel, C. Magen, Phys. Rev. B. 86 (2012) 14403.

[45] A.M. Pereira, J.B. Sousa, J.P. Araujo, P.A. Algarabel, L. Morellon, C. Marquina, M.R. Ibarra, Phys. Rev. B. 77 (2008) 134404.

[46] M. Han, J. Appl. Phys. 95 (2004) 6945.

[47] A. P. Ring, H.L. Ziegler, T. Lograsso, D. Schlagel, J.E. Snyder, D.C. Jiles, J. Appl. Phys. 99 (2006) 08R104. [48] Y. Mudryk, A.P. Holm, K.A. Gschneidner Jr., V.K. Pecharsky, Phys. Rev. B. 72 (2005) 64442.

[49] J.J. Rhyne, S. Legvold, Phys. Rev. 138 (1965).

[50] L. Morellon, C. Ritter, C. Magen, P. Algarabel, M. Ibarra, Phys. Rev. B. 68 (2003) 24417.

[51] A. Pires, J. Belo, A. Lopes, I. Gomes, L. Morellón, C. Magen, P. Algarabel, M. Ibarra, A. Pereira, J. Araújo, Entropy. 16 (2014) 3813.

[52] D. Paudyal, Y. Mudryk, V.K. Pecharsky, K. a. Gschneidner, Phys. Rev. B. 84 (2011) 14421.

[53] D. Paudyal, V. Pecharsky, K. Gschneidner, B. Harmon, Phys. Rev. B. 73 (2006) 144406.

[54] P. Kronmuller, H. and S., Handbook of Magnetism and Advanced Magnetic Materials, Fundamentals And 
Theory, 2007.

[55] C. Ritter, L. Morellon, P.A. Algarabel, C. Magen, M.R. Ibarra, Phys. Rev. B. 65 (2002) 944051. 


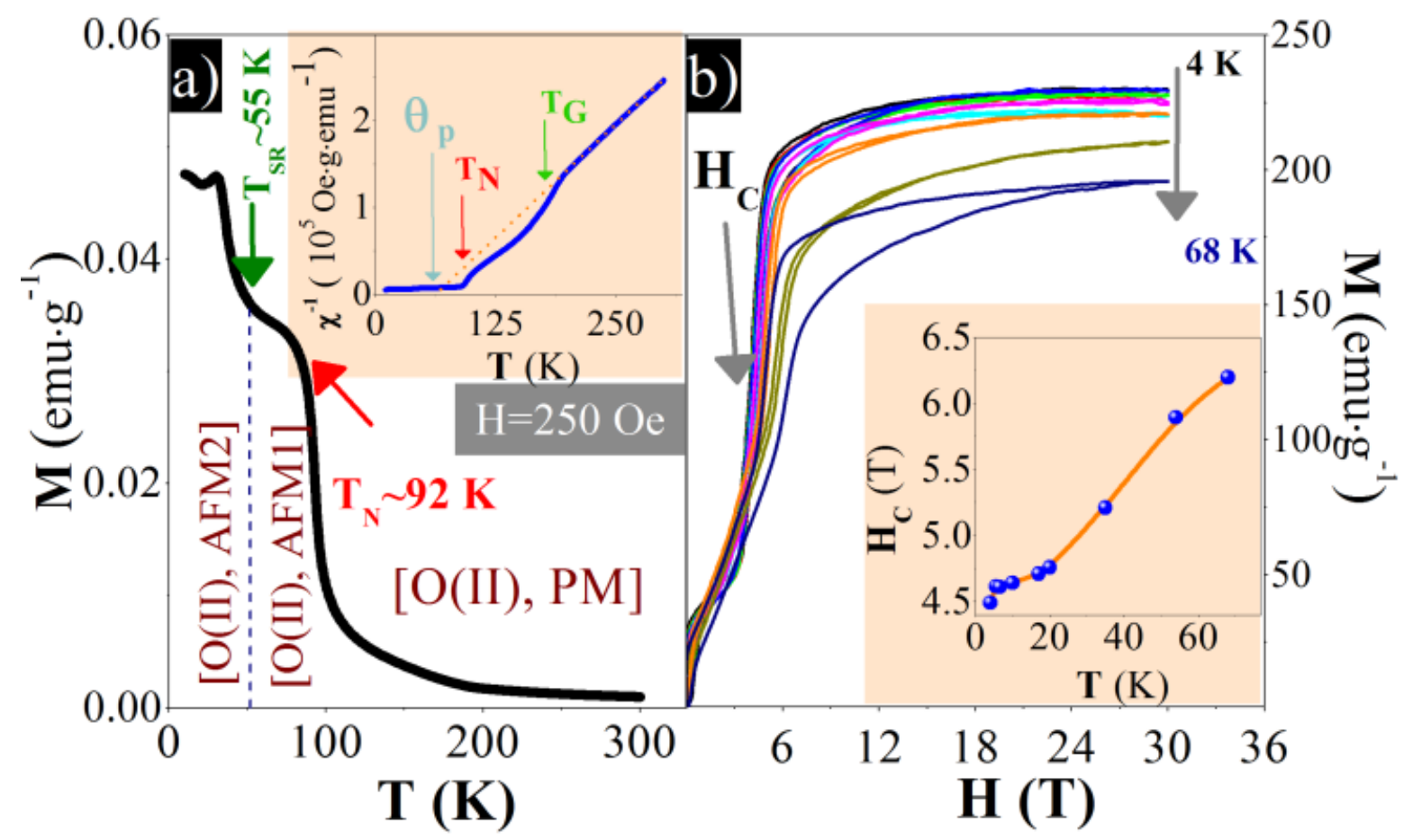

Figure 1: a) Temperature dependence of $\mathrm{Tb}_{5} \mathrm{Ge}_{4}$ magnetization measured with applied field: $\mathrm{H}$ $=250$ Oe. Inset: Reciprocal susceptibility versus temperature. b) Magnetization as a function of magnetic field in the $[0-30] \mathrm{T}$ range for a $[4,68] \mathrm{K}$ temperature interval. The critical magnetic field $\left(\mathrm{H}_{\mathrm{C}}\right)$ value versus temperature is displayed in the inset. 

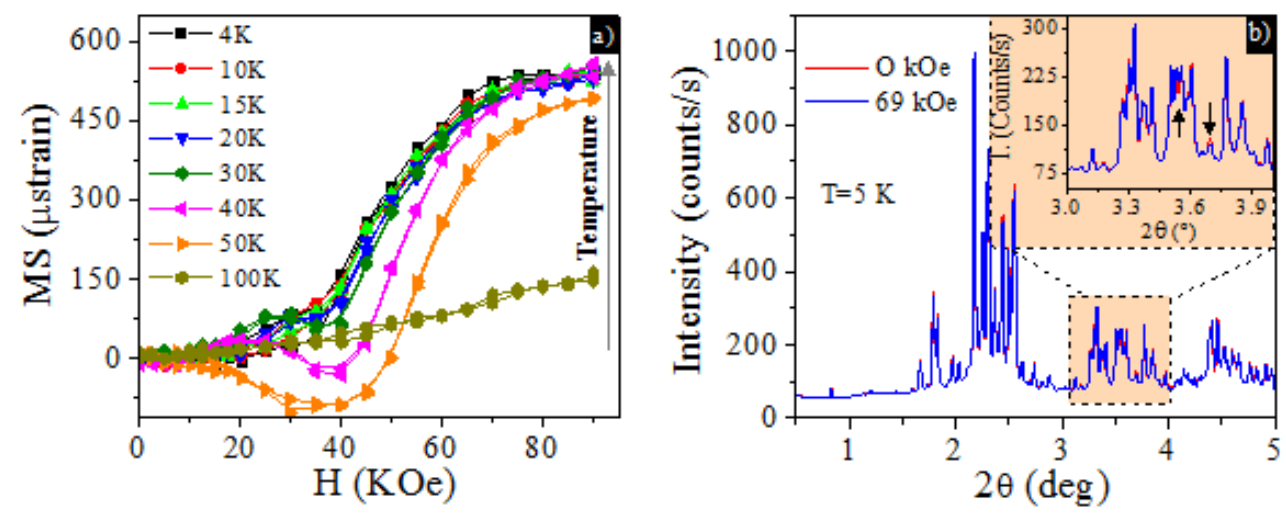

Figure 2: a) $\mathrm{Tb}_{5} \mathrm{Ge}_{4}$ magnetostriction dependence on the applied magnetic field (up to $90 \mathrm{kOe}$ ) in the temperature range of $5-100 \mathrm{~K}$ for $\mathrm{Tb}_{5} \mathrm{Ge}_{4}$. b) $\mathrm{Tb}_{5} \mathrm{Ge}_{4}$ High resolution X-ray synchrotron spectrum extracted under $\mathrm{H}_{\text {app }}=0$ and $69 \mathrm{kOe}$ at $\mathrm{T}=5 \mathrm{~K}$ with $\lambda=0.1066 \AA$ wavelength $\mathrm{x}$-ray radiation. 


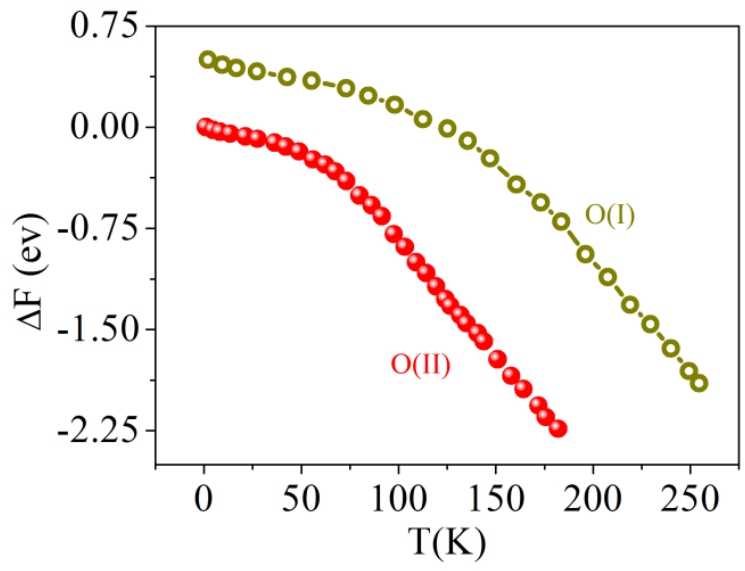

Figure 3: Temperature dependence of the Free energies of O(II) (red and closed dots, lower curve) and $\mathrm{O}(\mathrm{I})$ (green and empty dots, upper curve) structures for $\mathrm{Tb}_{5} \mathrm{Ge}_{4}$ compound. 


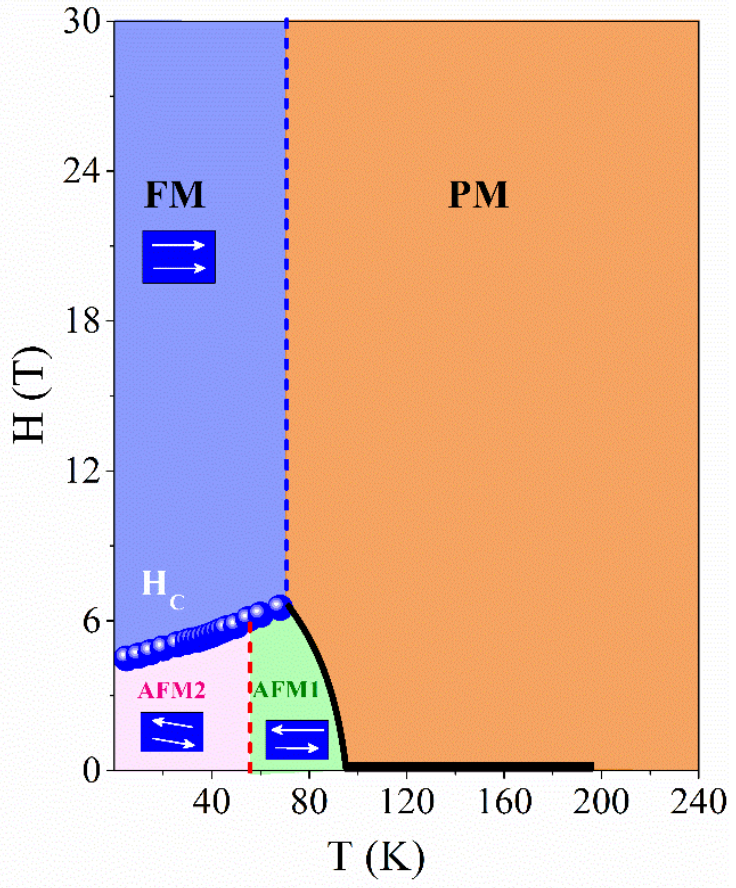

Figure 4: a) $(\mathrm{H}, \mathrm{T})$ phase diagram in the temperature range of $5-200 \mathrm{~K}$ for $\mathrm{Tb}_{5} \mathrm{Ge}_{4}$ compound. 